\title{
Genome-wide analysis of constitutional DNA methylation in familial melanoma
}

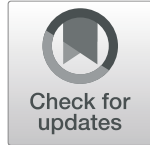

\author{
Catarina Salgado ${ }^{1}$ (D) Nelleke Gruis ${ }^{1}$ (D), BIOS Consortium, Bastiaan T. Heijmans ${ }^{2}$, Jan Oosting ${ }^{3+}$ (D) and \\ Remco van Doorn ${ }^{1 *}$ (1)
}

\begin{abstract}
Background: Heritable epigenetic alterations have been proposed as an explanation for familial clustering of melanoma. Here we performed genome-wide DNA methylation analysis on affected family members not carrying pathogenic variants in established melanoma susceptibility genes, compared with healthy volunteers.

Results: All melanoma susceptibility genes showed the absence of epimutations in familial melanoma patients, and no loss of imprinting was detected. Unbiased genome-wide DNA methylation analysis revealed significantly different levels of methylation in single CpG sites. The methylation level differences were small and did not affect reported tumour predisposition genes.
\end{abstract}

Conclusion: Our results provide no support for heritable epimutations as a cause of familial melanoma.

Keywords: Epimutation, Loss of imprinting, DNA methylation, Familial melanoma

\section{Introduction}

Cutaneous melanoma is an aggressive form of skin cancer with a propensity to metastasize, causing significant mortality and health care expenditure. Approximately $10 \%$ of patients diagnosed with melanoma have a positive family history for this malignancy. In familial or hereditary melanoma, multiple melanoma cases aggregate in several generations of a family, consistent with an autosomal dominant inheritance pattern. A subset of familial melanoma cases is caused by germline mutations in the established high penetrance melanoma predisposition genes $C D K N 2 A$ or $C D K 4$. Recently, pathogenic variants in the BAP1, TERT, POT1, TERF2IP, ACD and MITF genes have been identified as a cause of familial melanoma. Several candidate melanoma susceptibility genes including POLE, GOLM1 and EBF3 have been reported [1-3]. However, in more than half of affected families, the cause of melanoma predisposition

\footnotetext{
* Correspondence: rvandoorn@lumc.nl

†Jan Oosting and Remco van Doorn contributed equally to this work.

'Department of Dermatology, Leiden University Medical Center, Leiden, PO Box 9600, 2300 RC Leiden, The Netherlands

Full list of author information is available at the end of the article
}

remains to be resolved despite much research effort. For this reason, the attention has turned to different mechanisms of inheritance including heritable epigenetic alterations. Clarifying the genetic basis of familial melanoma is clinically relevant as it would allow for genetic testing, risk estimation and targeted clinical surveillance of patients at high risk of melanoma.

Epimutations have been defined as heritable changes in gene activity due to DNA modifications, not encompassing changes in the DNA sequence itself [4]. It has been postulated to constitute an alternative mechanism to genetic mutation for cancer predisposition and commonly refers to constitutional promoter CpG island hypermethylation in all somatic cells of an individual [5]. The best-described example in cancer is hereditary nonpolyposis colorectal cancer (HNPCC, Lynch syndrome) where cases not affected by inactivating mutations in DNA mismatch repair genes were found to be caused by heritable promoter hypermethylation of the $M L H 1$ gene [6-8]. Epimutations have been classified as primary, occurring in the absence of an underlying DNA sequence alteration, and secondary, when a genetic mutation 
triggers the occurrence of an epigenetic modification [9]. Secondary epimutations in the MSH2 and DAPK1 gene have been identified in HNPCC and familial chronic lymphocytic leukemia, respectively [10-12].

Genomic imprinting causes certain genes to be silenced by DNA and histone methylation in a parent of originspecific manner, ensuring proper expression during development. Loss of imprinting is a distinct epigenetic mechanism of disease, associated with deregulated gene expression that can be implicated in cancer development [13]. The association between loss of imprinting at the IGF2-H19 locus at chromosome $11 \mathrm{p} 15.5$ and predisposition to Wilms tumour is an example of this epigenetic mechanism [14].

In familial melanoma, we and others have shown the absence of epimutation of the CDKN2A gene, the major high penetrance melanoma susceptibility gene $[15,16]$. A previous study analysed methylation of 14 cancerrelated genes in blood DNA from melanoma-prone family members. This analysis revealed no constitutional promoter hypermethylation, but reduced methylation of the TNFRSF10C promoter [17].

In this study, we aim to identify heritable epigenetic alterations that might account for familial clustering of melanoma in families where no genetic variants in established or candidate melanoma susceptibility genes were found. To this end, a genome-wide methylation analysis of peripheral blood DNA from patients with familial melanoma was performed. We assessed promoter hypermethylation of recently identified melanoma susceptibility genes and loss of imprinting and performed an unbiased analysis of hypermethylated $\mathrm{CpG}$ sites and regions.

\section{Results and discussion}

Patients from 5 Dutch families with at least 3 melanoma cases in different generations were selected for this study

Table 1 Clinical characteristics of patients/families involved in the genome-wide analysis

\begin{tabular}{|c|c|c|c|c|c|}
\hline Family & $\begin{array}{l}\text { Number of CMM } \\
\text { affected members }\end{array}$ & $\begin{array}{l}\text { Patient } \\
\text { number }\end{array}$ & $\begin{array}{l}\text { Degree } \\
\text { of kinship }\end{array}$ & $\begin{array}{l}\text { Age at } \\
\text { melanoma } \\
\text { diagnosis }\end{array}$ & $\begin{array}{l}\text { Age at } \\
\text { DNA } \\
\text { collection }\end{array}$ \\
\hline \multirow[t]{2}{*}{ I } & 9 & I_1 & 2nd & 62 & 76 \\
\hline & & $1 \_2$ & & $\begin{array}{l}34,34,46, \\
48^{\mathrm{a}}\end{array}$ & 53 \\
\hline \multirow[t]{2}{*}{$\|$} & 5 & ॥_1 & $3 r d$ & 41 & 52 \\
\hline & & II_2 & & 52 & 56 \\
\hline \multirow[t]{2}{*}{ III } & 4 & III_1 & $2 n d$ & $51,57^{\mathrm{a}}$ & 68 \\
\hline & & III_2 & & 28 & 33 \\
\hline \multirow[t]{2}{*}{ IV } & 3 & IV_1 & $3 r d$ & 34 & 56 \\
\hline & & IV_2 & & 35 & 43 \\
\hline \multirow[t]{2}{*}{ V } & 4 & $V_{-} 1$ & $1 \mathrm{st}$ & 49 & 55 \\
\hline & & V_2 & & 30 & 25 \\
\hline
\end{tabular}

${ }^{a}$ Multiple primary melanomas diagnosed
(Table 1, pedigrees in Supplementary Figure 1). The presence of pathogenic gene variants in all currently established and candidate high penetrance melanoma susceptibility genes was assessed in all included cases using whole-genome sequencing. No germline mutations were found in these genes. To examine DNA methylation, DNA from peripheral blood of 2 affected members of each family $(n=10)$ was subjected to $450 \mathrm{~K}$ Illumina arrays interrogating over 450,000 CpG sites (namely 483,891 probes after quality control) covering $99 \%$ of human genes following bisulfite conversion [18]. For comparative analysis, we could make use of DNA methylation data obtained from peripheral blood samples of a reference group of 1000 healthy Dutch individuals included in the Biobank-based Integrative Omics Studies (BIOS) consortium analysed using similar $450 \mathrm{~K}$ arrays (raw data available from the European Genome-phenome Archive (EGA) under accession EGAS00001001077). All samples were compared individually to the reference group, while taking multiple testing into account using Bonferroni correction.

First, we analysed the presence of promoter hypermethylation in the CDKN2A, CDK4, BAP1, TERT, POT1, TERF2IP, ACD, MBD4, POLH, MITF, MC1R, POLE, EBF3 and GOLM1 melanoma susceptibility genes. All CpG sites designated by a probe located across the entire sequence of these melanoma susceptibility genes in the familial melanoma samples were compared with reference samples. No significant difference ( $\geq \beta$ value average $\pm 5.65 \mathrm{SD})$ in methylation level, hypermethylation nor hypomethylation was found at any of these genes (Fig. 1).

The methylation status of imprinted genes in familial melanoma patients was then investigated. We checked all CpG sites in the entire gene sequence of all imprinted genes in humans (http://www.geneimprint.com/site/ genes-by-species, accessed August 2019) interrogated by the arrays. The methylation levels of 4790 interrogated CpG sites located at these imprinted gene loci did not differ significantly ( $\geq \beta$ value average $\pm 5.65 \mathrm{SD}$ ) from the BIOS reference samples (Supplementary Figure 2). Since the regulation of imprinted genes is largely dependent on methylation levels, and there is no significant difference in any of the familial melanoma patients compared to BIOS, we conclude that there was no indication of loss of imprinting.

Following analysis of candidate genes, we performed an agnostic genome-wide analysis by comparing DNA methylation of all interrogated CpG sites in the familial melanoma patients with those in healthy subjects. We considered as potential epimutations $\mathrm{CpG}$ sites located in gene promoters (using probes assigned to promoter regions according to annotation provided by Illumina) with significantly aberrant methylation levels in both members of an affected family compared to BIOS 


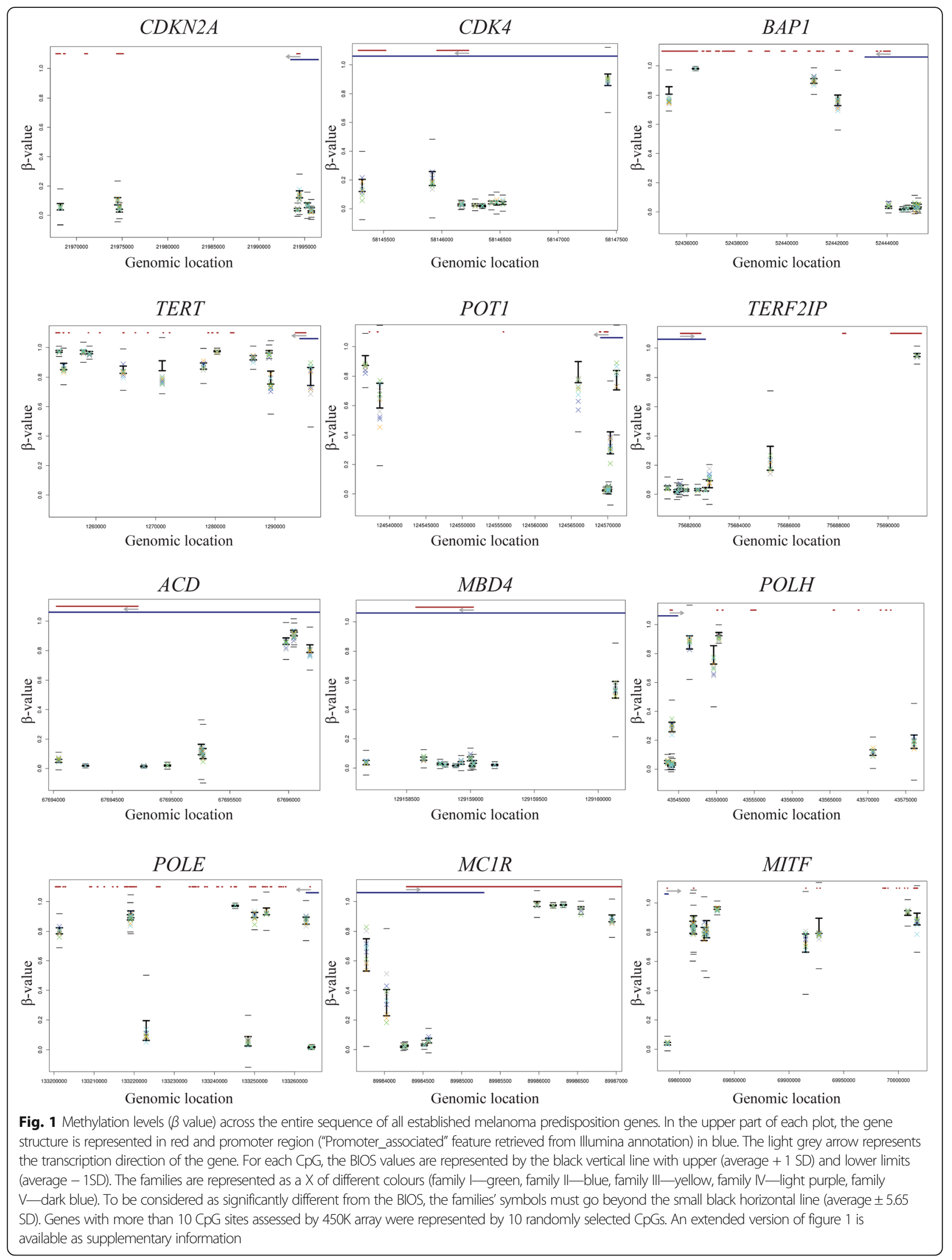


control samples. Since all reported cancer-predisposing epimutations were cases of constitutional promoter hypermethylation, we focused our analysis on this type of epigenetic event. All CpGs in promoters were assessed. Probes interrogating CpG sites lost due to single nucleotide variants, as identified using wholegenome sequence data, were not included in the analysis of the affected samples. We identified 6 single CpGs in gene promoters with significantly higher $\beta$ values in both affected members of a melanoma family compared to healthy controls (Fig. 2, Table 2, Supplementary Table 1). In healthy controls, these $\mathrm{CpG}$ sites showed low average $\beta$ values consistent with the absence of methylation. The CpG sites in the RABGGTB, SND1, SCAF11, ZNF638, THAP1 and SFSWAP genes showed significantly higher $\Delta \beta$ values in both members of multiple families.

We assessed the methylation of contiguous interrogated CpG sites, and for all 6 cases, the hypermethylation was observed exclusively in the single identified CpG site, with neighbouring $\mathrm{CpGs}$ not showing significantly higher methylation levels. None of these genes have been reported as cancer predisposition genes by Rahman [19]. Only one of the CpGs is located in a cancer-related gene,
SND1, according to Cancer Gene Census (http://cancer. sanger.ac.uk/census, accessed August 2019). This gene has no reported role in melanoma and functions as a gene fusion partner in certain malignancies. Given the established genetic heterogeneity, it is unlikely that the same epimutation would cause melanoma susceptibility in all 5 families. Together with the information about the function of the genes (Supplementary Table 1), we conclude that the identified hypermethylated CpG sites in these families do not appear to constitute plausible pathogenic high penetrance epimutation events.

Additionally, we evaluated CpG sites with significantly lower methylation levels in familial melanoma than in healthy control samples and found 35 hypomethylated CpGs in both members of a family (Supplementary Table 2). Fifteen CpG sites showed hypomethylation in all 5 families, suggestive of a batch effect as has been described for $450 \mathrm{k}$ methylation arrays [20]. Of the 35 hypomethylated CpG sites, only 2 were located in established cancer-related genes: BRCA1, an established breast and ovarian cancer susceptibility gene, and ROS1, encoding a receptor tyrosine kinase with a possible oncogenic role in melanoma [21]. For both genes, a

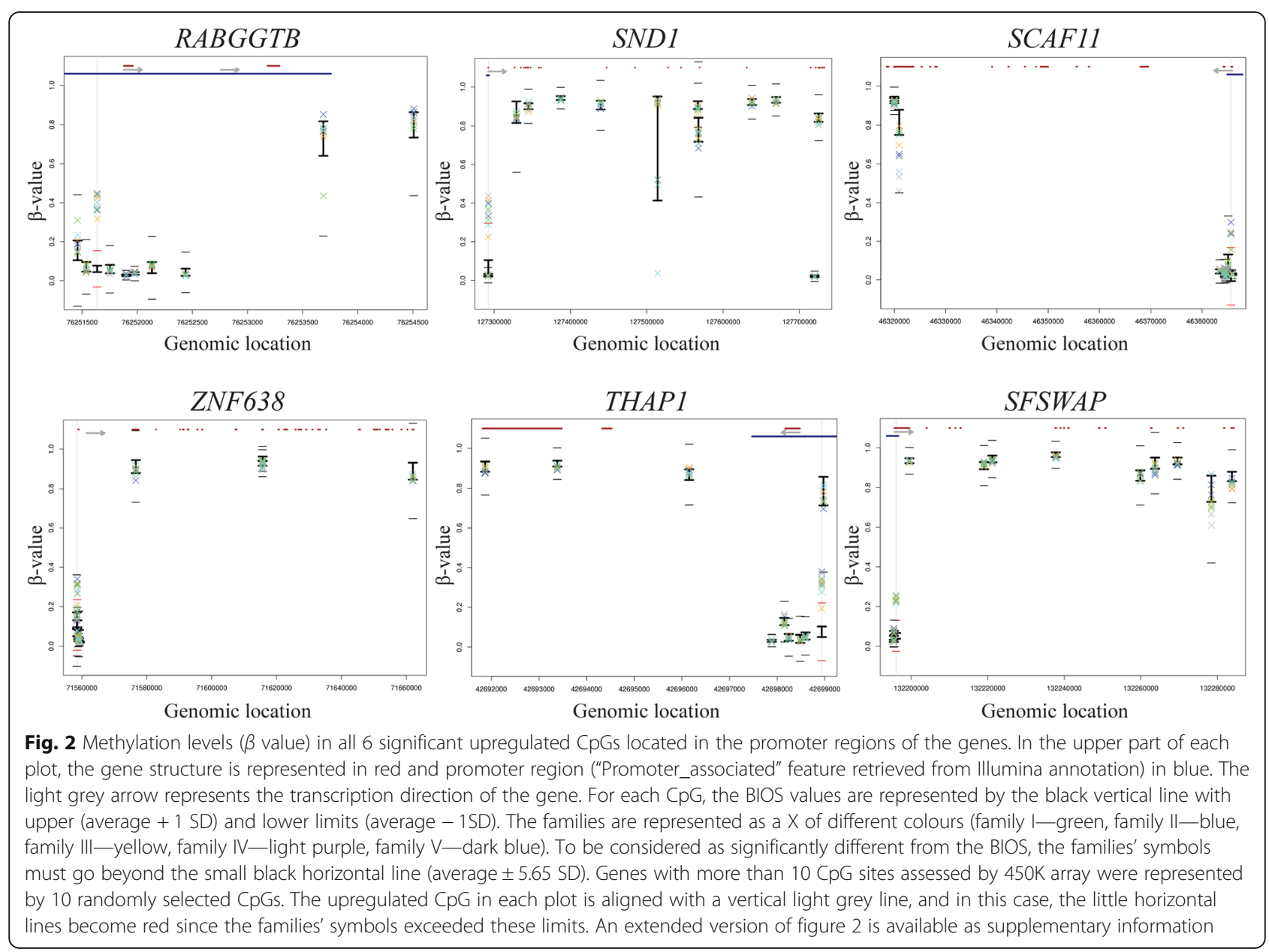


Table 2 Methylation levels ( $\Delta \beta$ value) in all 6 significant upregulated CpGs in all subjects $(n=10)$

\begin{tabular}{|c|c|c|c|c|c|c|c|c|c|c|c|c|}
\hline \multirow[t]{3}{*}{ CpG ID } & \multirow[t]{3}{*}{ Gene ID } & \multirow{3}{*}{$\begin{array}{l}\text { BIOS } \\
\text { control cases } \\
\beta \text { value }^{a}\end{array}$} & \multicolumn{2}{|c|}{ Family I } & \multicolumn{2}{|c|}{ Family II } & \multicolumn{2}{|c|}{ Family III } & \multicolumn{2}{|c|}{ Family IV } & \multicolumn{2}{|c|}{ Family V } \\
\hline & & & I_1 & I_2 & II_1 & II_2 & III_1 & III_2 & IV_1 & IV_2 & $V_{-} 1$ & V_2 \\
\hline & & & \multicolumn{10}{|c|}{$\Delta \beta$ value $^{\mathrm{b}}$} \\
\hline cg21812670 & RABGGTB & 0.06 & 0.30 & 0.38 & 0.34 & 0.30 & 0.36 & 0.26 & 0.39 & 0.32 & 0.39 & 0.30 \\
\hline cg26642667 & SND1 & 0.06 & 0.25 & 0.30 & 0.33 & 0.22 & 0.35 & 0.16 & 0.37 & 0.29 & 0.34 & 0.27 \\
\hline cg04385631 & SCAF11 & 0.02 & 0.14 & 0.00 & 0.00 & 0.22 & 0.00 & 0.22 & 0.23 & 0.00 & 0.22 & 0.28 \\
\hline cg21843755 & ZNF638 & 0.11 & 0.16 & 0.21 & 0.18 & 0.07 & 0.20 & 0.10 & 0.21 & 0.16 & 0.23 & 0.21 \\
\hline cg03301282 & THAP1 & 0.08 & 0.26 & 0.23 & 0.20 & 0.27 & 0.25 & 0.12 & 0.29 & 0.27 & 0.30 & 0.24 \\
\hline cg02470959 & SFSWAP & 0.05 & 0.18 & 0.20 & 0.18 & 0.16 & 0.19 & 0.17 & 0.21 & 0.21 & 0.20 & 0.17 \\
\hline
\end{tabular}

${ }^{\text {a }}$ Represents the average $\beta$ value for the 1000 BIOS controls at each CpG site

${ }^{b}$ Represents the difference between BIOS average $\beta$ value and patient $\beta$ value at each CpG site

single CpG site in the promoter demonstrated significantly lower methylation levels, with normal methylation of neighbouring $\mathrm{CpG}$ sites assessed by $450 \mathrm{~K}$ array. For $B R C A 1$ and for ROS1, the CpG site was not part of a predicted transcription factor binding motif [22]. Hypomethylation of the BRCA1 gene promoter has never been associated with transcriptional downregulation, and therefore, reduced methylation of this single CpG site in the $B R C A 1$ gene promoter is unlikely to have pathogenic significance. Expression of the ROS1 oncogene is not known to be regulated by promoter methylation, but high expression has been associated with histone modifications and EZH2 repression [23]. $\beta$ values for the single CpG site in the distal promoter of the ROS1 gene were 0.87 in control samples and approximately 0.65 in familial melanoma DNA samples. We consider it possible, but unlikely that lower methylation levels of a single CpG site in the distal promoter of ROS1 would cause familial melanoma. Similar to the finding of TNFRSF10C hypomethylation in familial melanoma patients from the USA, which we could not detect in our patients, this finding might be analysed in a large number of melanoma families [17].

Since regions containing multiple CpG sites in promoters commonly work as units of transcriptional regulation, we additionally tried to identify differentially methylated regions. For this, we evaluated the average of all probes assigned to promoters for each gene comparing familial melanoma and healthy control samples. The annotation of the $450 \mathrm{~K}$ array contains 13,715 genes with CpG probes assigned to promoters. The promoter of one gene $(C C N I)$ showed significant higher methylation levels in 4 families, while promoters of the CD47 and USP46 genes had slightly higher methylation levels in 1 family each. Although statistically significant, the averaged promoter methylation level ( $\beta$ value) differences were minor, which does not support a relevant effect.

In this study, we analysed the possible occurrence of epimutations and loss of imprinting in familial melanoma using a genome-wide approach. A strength of the study is the selection of DNA samples from families with many affected members in multiple generations where no genetic cause could be identified and the availability of methylation data from a large cohort of 1000 Dutch healthy individuals for comparative analysis. There are some limitations to this study; the number of analysed families is small, and our results do not exclude the possibility that pathogenic epimutations might occur in a small proportion of melanoma families. Secondly, the $450 \mathrm{~K}$ arrays interrogate $\mathrm{CpG}$ sites in almost all gene promoters, but do not cover all potentially regulatory sequences. In addition, we analysed blood DNA for the occurrence of epimutations, but certain epimutations might occur only in specific cell types in a mosaic state. In these patients with familial melanoma, we have not identified promoter hypermethylation of any melanoma predisposition gene, cancer predisposition gene or tumour suppressor gene. We have been able to determine several DNA methylation events that are candidate epimutations, methylation events shared by multiple members of a family that were not identified in healthy volunteers. However, it is not clear if the observed methylation alterations in these single CpG sites impact on expression of the respective genes. Based on the function of the genes and the fact that we did not identify a differentially methylated region, but only a single CpG site, we consider it is not plausible that any of the DNA methylation alterations that were detected constitute the cause of melanoma predisposition in these families. Moreover, given the established genetic heterogeneity, it is unlikely that the same epimutation would cause melanoma susceptibility in all 5 families. Therefore, we consider the observed $\mathrm{CpG}$ sites with higher and lower detected methylation levels to represent rare variations with no pathogenic significance or possibly the result of batch array effects. In summary, our results of genome-wide analysis provide little or no support for a role of heritable DNA methylation alterations as a cause of familial melanoma. 


\section{Materials and methods}

We selected 5 unrelated Dutch families with 3 or more melanoma cases in multiple generations and tested negative for germline mutations in the established high penetrance melanoma susceptibility genes CDKN2A, CDK4, BAP1, TERT, POT1, TERF2IP, ACD and MITF by next-generation sequencing (Fig. 1). Some patients had developed multiple melanomas. The majority of the melanomas were of the superficial spreading or nodular subtypes. The study was approved by the Leiden University Medical Center institutional ethical committee and was conducted according to the Declaration of Helsinki principles. DNA from 2 affected members from 5 families was isolated from whole blood samples. DNA was bisulfite-converted using the EZ DNA methylation kit (Zymo Research, D5001) and hybridized to Illumina $450 \mathrm{~K}$ arrays (Illumina). The reference group encompassed 1000 whole blood DNA samples of healthy individuals included in the Biobank-based Integrative Omics Studies (BIOS) Consortium analysed with Illumina 450K arrays under similar conditions [24]. The median age of patients during blood sampling was 54 years, and for the 1000 healthy controls, it was 55 years. Sample quality control was performed using MethylAid [25], and probes with a high detection $P$ value $(>0.01$ ), probes with a low bead count ( $<3$ beads) and probes with a low success rate (missing in $>95 \%$ of the samples) were set to missing. Subsequently, imputation [26] was performed to impute the missing values. Functional normalization, as implemented in the minfi package, was used on a random subset of 1000 samples together with the melanoma samples [27]. A detailed description of the $450 \mathrm{~K}$ DNA methylation pre-processing steps is available from the https://molepi.github.io/DNAmArray_workflow/. Sample specific aberrant melanoma CpGs were detected using a $t$ test for comparing a single melanoma case to the 1000 BIOS controls [28]. In order to control for the number of tests, a very stringent cut-off, $1.03 \times 10^{-9}$ $(0.01 /($ number of probes on array $\times 2))$, was used. After the bioinformatic analysis, a set of 13 hypermethylated CpGs and 164 hypomethylated CpG sites was obtained. The list of significant CpGs was further reduced by only considering significant co-segregating CpGs with an absolute $\beta$ value difference of 0.2 when compared to BIOS controls $(\Delta \beta$ value $\geq 0.2$ in 2 members of at least one family). To be considered as a putative epimutation, a CpG should meet the following criteria. CpG probes on chromosome $\mathrm{X}$ were excluded (as they reflect $\mathrm{X}$ chromosome inactivation in females). Only CpGs in promoter regions (retrieved from Illumina annotation for gene promoters, "promoter_associated in regulatory_feature_group field") of the genes were selected. Both members of a family were required to harbor the hypo/ hypermethylation, since we are looking at high penetrance epigenetic events. If there is a single nucleotide variant (SNV) within a window of $100 \mathrm{bp}$ around the $\mathrm{CpG}$ (that can either influence/impair the probe binding or reveal the presence of a genetic variant around the epigenetically altered $\mathrm{CpG}$, that in this case would be the so-called second epimutation), this $\mathrm{CpG}$ must be excluded. The SNV data were retrieved from dbSNP Release 153. This resulted in 6 hypermethylated CpGs and 35 hypomethylated CpGs, which were compared with lists of cancer-related genes according to Cancer Gene Census (http://cancer.sanger.ac.uk/census, accessed August 2019) and cancer predisposition genes suggested by Rahman [19]. We have checked whether some CpG sites of interest were part of predicted transcription factor binding motifs using the TFBIND tool [22]. We also aimed at identifying differentially methylated regions. For that, we assessed the probes assigned for promoter regions according to the annotation of $450 \mathrm{~K}$ array. There were 13,715 genes with probes assigned to promoters. On average, each of these promoters contained 6.7 probes. We averaged all the probes assigned for each gene promoter and compared with the average of the same promoter in BIOS controls.

\section{Supplementary information}

Supplementary information accompanies this paper at https://doi.org/10. 1186/s13148-020-00831-7.

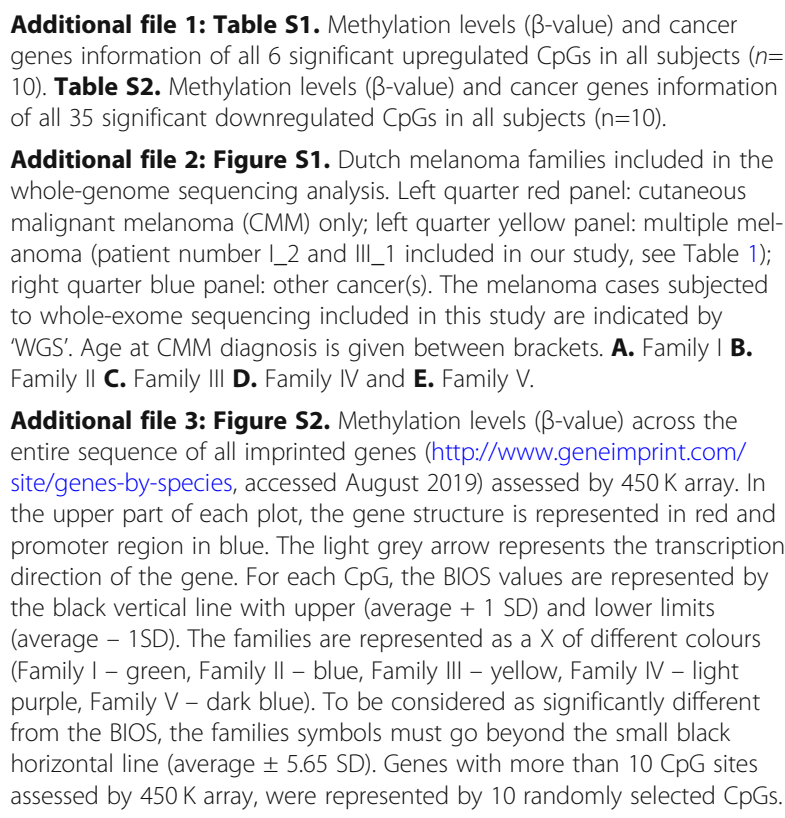

Additional file 2: Figure S1. Dutch melanoma families included in the whole-genome sequencing analysis. Left quarter red panel: cutaneous malignant melanoma (CMM) only; left quarter yellow panel: multiple melanoma (patient number I_2 and III_1 included in our study, see Table 1); right quarter blue panel: other cancer(s). The melanoma cases subjected to whole-exome sequencing included in this study are indicated by 'WGS'. Age at CMM diagnosis is given between brackets. A. Family I B. Family II C. Family III D. Family IV and E. Family V.

Additional file 3: Figure S2. Methylation levels ( $\beta$-value) across the entire sequence of all imprinted genes (http://www.geneimprint.com/ site/genes-by-species, accessed August 2019) assessed by $450 \mathrm{~K}$ array. In the upper part of each plot, the gene structure is represented in red and promoter region in blue. The light grey arrow represents the transcription direction of the gene. For each CpG, the BIOS values are represented by the black vertical line with upper (average +1 SD) and lower limits (average - 1SD). The families are represented as a X of different colours (Family I - green, Family II - blue, Family III - yellow, Family IV - light purple, Family $V$ - dark blue). To be considered as significantly different from the BIOS, the families symbols must go beyond the small black horizontal line (average \pm 5.65 SD). Genes with more than $10 \mathrm{CpG}$ sites assessed by $450 \mathrm{~K}$ array, were represented by 10 randomly selected CpGs.

\section{Abbreviations}

BIOS Consortium: Biobank-based Integrative Omics Studies; EGA: European Genome-phenome Archive; HNPCC: Hereditary nonpolyposis colorectal cancer; SNV: Single nucleotide variant 


\section{Acknowledgements}

We thank AG Jochemsen and Mijke Visser for useful discussions. We acknowledge MELGEN, a Marie-Curie Consortium for all the scientific support.

\section{Authors' contributions}

CS analysed, filtered and interpreted the bioinformatic data and wrote the manuscript. NG and $\mathrm{BH}$ were involved in scientific and technical discussions. $\mathrm{BH}$ gave access to $\mathrm{BIOS}$ consortium data. JO performed the bioinformatic analysis of patient data and healthy Dutch individuals from BIOS. RvD was responsible for the conception and design of the work. All authors read, revised and approved the final manuscript.

\section{Funding}

This project was supported by the European Union's Horizon 2020 research and innovation programme under grant agreement no. 641458 and the Dutch Cancer Foundation grant UL2012-5489. This research was also financially supported by BBMRI-NL, a Research Infrastructure financed by the Dutch government (NWO, numbers 184.021.007 and 184.033.111).

\section{Availability of data and materials}

The dataset supporting the conclusions of this article, Biobank-based Integrative Omics Studies (BIOS) consortium, is available in the European Genomephenome Archive (EGA) under accession EGAS00001001077 (https://www. ebi.ac.uk/ega/studies/EGAS00001001077)

\section{Ethics approval and consent to participate}

The study was approved by the Leiden University Medical Center institutional ethical committee and was conducted according to the Declaration of Helsinki principles.

\section{Consent for publication}

Not applicable.

\section{Competing interests}

The authors declare that they have no competing interests.

\section{Author details}

'Department of Dermatology, Leiden University Medical Center, Leiden, PO Box 9600, 2300 RC Leiden, The Netherlands. ${ }^{2}$ Molecular Epidemiology, Department of Biomedical Data Sciences, Leiden University Medical Center, Leiden, The Netherlands. ${ }^{3}$ Department of Pathology, Leiden University Medical Center, Leiden, The Netherlands.

\section{Received: 31 October 2019 Accepted: 20 February 2020}

Published online: 06 March 2020

\section{References}

1. Teerlink CC, Huff C, Stevens J, Yu Y, Holmen SL, Silvis MR, et al. A nonsynonymous variant in the GOLM1 gene in cutaneous malignant melanoma. J Natl Cancer Inst. 2018:110(12):1380-5.

2. Aoude LG, Heitzer E, Johansson P, Gartside M, Wadt K, Pritchard AL, et al. POLE mutations in families predisposed to cutaneous melanoma. Familial Cancer. 2015;14(4):621-8

3. Chatterjee A, Stockwell PA, Ahn A, Rodger EJ, Leichter AL, Eccles MR. Genome-wide methylation sequencing of paired primary and metastatic cell lines identifies common DNA methylation changes and a role for EBF3 as a candidate epigenetic driver of melanoma metastasis. Oncotarget. 2017; 8(4):6085-101.

4. Holliday R. The inheritance of epigenetic defects. Science. 1987;238(4824): 163-70.

5. Hitchins MP. Constitutional epimutation as a mechanism for cancer causality and heritability? Nat Rev Cancer. 2015;15(10):625-34

6. Suter CM, Martin DI, Ward RL. Germline epimutation of MLH1 in individuals with multiple cancers. Nat Genet. 2004;36(5):497-501.

7. Hitchins MP, Rapkins RW, Kwok CT, Srivastava S, Wong JJ, Khachigian LM, et al. Dominantly inherited constitutional epigenetic silencing of MLH1 in a cancer-affected family is linked to a single nucleotide variant within the 5'UTR. Cancer Cell. 2011;20(2):200-13.
8. Hitchins MP, Wong JJ, Suthers G, Suter CM, Martin DI, Hawkins NJ, et al. Inheritance of a cancer-associated MLH1 germ-line epimutation. N Engl J Med. 2007;356(7):697-705.

9. Horsthemke B. Epimutations in human disease. Curr Top Microbiol Immunol. 2006;310:45-59.

10. Chan TL, Yuen ST, Kong CK, Chan YW, Chan AS, Ng WF, et al. Heritable germline epimutation of $\mathrm{MSH} 2$ in a family with hereditary nonpolyposis colorectal cancer. Nat Genet. 2006:38(10):1178-83.

11. Ligtenberg MJ, Kuiper RP, Chan TL, Goossens M, Hebeda KM, Voorendt M, et al. Heritable somatic methylation and inactivation of MSH2 in families with Lynch syndrome due to deletion of the $3^{\prime}$ exons of TACSTD1. Nat Genet. 2009;41(1):112-7.

12. Raval A, Tanner SM, Byrd JC, Angerman EB, Perko JD, Chen SS, et al. Downregulation of death-associated protein kinase 1 (DAPK1) in chronic lymphocytic leukemia. Cell. 2007;129(5):879-90.

13. Uribe-Lewis S, Woodfine K, Stojic L, Murrell A. Molecular mechanisms of genomic imprinting and clinical implications for cancer. Expert Rev Mol Med. 2011;13:e2

14. Moulton T, Crenshaw T, Hao Y, Moosikasuwan J, Lin N, Dembitzer F, et al. Epigenetic lesions at the $\mathrm{H} 19$ locus in Wilms' tumour patients. Nat Genet. 1994;7(3):440-7.

15. Erlandson A, Appelqvist F, Enerback C. Epigenetic mutations in CDKN2A in western Swedish families with hereditary malignant melanoma. Mol Med Rep. 2008;1(1):89-91.

16. van Doorn R, Zoutman WH, Gruis NA. Absence of germline epimutation of the CDKN2A gene in familial melanoma. J Invest Dermatol. 2009:129(3):781-4.

17. Hyland PL, Burke LS, Pfeiffer RM, Rotunno M, Sun D, Patil P, et al. Constitutional promoter methylation and risk of familial melanoma. Epigenetics. 2014;9(5):685-92.

18. Sandoval J, Heyn H, Moran S, Serra-Musach J, Pujana MA, Bibikova M, et al. Validation of a DNA methylation microarray for $450,000 \mathrm{CpG}$ sites in the human genome. Epigenetics. 2011;6(6):692-702.

19. Rahman N. Realizing the promise of cancer predisposition genes. Nature. 2014;505(7483):302-8.

20. Price EM, Robinson WP. Adjusting for batch effects in DNA methylation microarray data, a lesson learned. Front Genet. 2018;9:83.

21. Wiesner T, He J, Yelensky R, Esteve-Puig R, Botton T, Yeh I, et al. Kinase fusions are frequent in Spitz tumours and spitzoid melanomas. Nat Commun. 2014:5:3116.

22. Tsunoda T, Takagi T. Estimating transcription factor bindability on DNA. Bioinformatics. 1999:15(7-8):622-30.

23. Shih $\mathrm{CH}$, Chang YJ, Huang WC, Jang TH, Kung HJ, Wang WC, et al. EZH2mediated upregulation of ROS1 oncogene promotes oral cancer metastasis. Oncogene. 2017:36(47):6542-54

24. Bonder MJ, Luijk R, Zhernakova DV, Moed M, Deelen P, Vermaat M, et al. Disease variants alter transcription factor levels and methylation of their binding sites. Nat Genet. 2017;49(1):131-8.

25. van Iterson M, Tobi EW, Slieker RC, den Hollander W, Luijk R, Slagboom PE, et al. MethylAid: visual and interactive quality control of large Illumina 450k datasets. Bioinformatics. 2014;30(23):3435-7.

26. Troyanskaya O, Cantor M, Sherlock G, Brown P, Hastie T, Tibshirani R, et al. Missing value estimation methods for DNA microarrays. Bioinformatics. 2001;17(6):520-5.

27. Fortin JP, Labbe A, Lemire M, Zanke BW, Hudson TJ, Fertig EJ, et al. Functional normalization of 450k methylation array data improves replication in large cancer studies. Genome Biol. 2014;15(12):503.

28. Crawford JR, Garthwaite PH. Single-case research in neuropsychology: a comparison of five forms of t-test for comparing a case to controls. Cortex. 2012:48(8):1009-16.

\section{Publisher's Note}

Springer Nature remains neutral with regard to jurisdictional claims in published maps and institutional affiliations. 